\title{
Innovative Thermobalancing therapy and Dr Allen's Device for the first time employ body energy to treat chronic prostatic diseases effectively
}

\author{
S. Allen(1)
}

Correspondence: info@

finetreatment.com

Fine Treatment, Oxford, UK

\begin{abstract}
Benign prostatic hyperplasia (BPH) and chronic prostatitis / chronic pelvic pain syndrome (CP/CPPS) are common chronic diseases. None of the available treatments are effective in the management of these diseases. The purpose of this study was to assess the efficacy of Thermobalancing therapy (TT) with therapeutic Dr Allen's Device (DATD) for the treatment of BPH and CP/CPPS. Retrospective analysis of 2 clinical studies employing 6-month $\pi$ for the treatment of BPH $(n=124)$ and CP/ CPPS $(n=45)$ was conducted. The efficacy of the $\Pi$ with DATD was assessed using validated and widely accepted parameters. Results were compared with corresponding control groups comprising of subjects who did not receive any treatment. In BPH treatment group, urinary symptoms decreased (14.3 to 4.7) and QoL improved (3.9 to 1.39). In CP/CPPS treatment group, pain score decreased (10.3 to 3.5) and QoL improved (8.1 to 2.9). The results in the 2 treatment groups were statistically significantly better than the results obtained for the corresponding control groups $(P<0.001)$. Innovative $\Pi T$ with DATD provides an effective treatment that uniquely employs body energy for the management of BPH and CP/CPPS.
\end{abstract}

Keywords: Chronic pelvic pain, Benign prostatic hyperplasia (BPH), Chronic prostatitis, Thermobalancing therapy and Dr Allen's Device, Body energy

\section{Introduction}

Benign prostatic hyperplasia (BPH) is one of the most common chronic disorders in elderly men, the incidence of which increases with age. Clinically, it is a non-malignant progressive proliferation of prostatic epithelial tissue and smooth muscles resulting in an increased prostate volume beyond the normal value of $20-30 \mathrm{~mL}$. According to an estimate, the age-related prevalence of BPH in the United States (US) ranged from 50 to $75 \%$ among men $>50$ years of age and to $80 \%$ among men $>70$ years of age, which corresponds to approximately 15 million men [1]. The age-related prevalence of $\mathrm{BPH}$ has been reported to be $8 \%$ in men aged about 40 years and it reaches $90 \%$ by 90 years of age [2]. Similar trends have been reported from different parts of the world

(c) The Author(s). 2020 Open Access This article is licensed under a Creative Commons Attribution 4.0 International License, which permits use, sharing, adaptation, distribution and reproduction in any medium or format, as long as you give appropriate credit to the original author(s) and the source, provide a link to the Creative Commons licence, and indicate if changes were made. The images or other third party material in this article are included in the article's Creative Commons licence, unless indicated otherwise in a credit line to the material. If material is not included in the article's Creative Commons licence and your intended use is not permitted by statutory regulation or exceeds the permitted use, you will need to obtain permission directly from the copyright holder. To view a copy of this licence, visit http://creativecommons.org/licenses/by/4.0/. 
[3]. Increases in prostate size exert pressure on the nearby organs and block the passage of urine from the urethra.

Thus, BPH is generally associated with a constellation of urinary symptoms that are divided into 2 major classes: (1) bladder storage symptoms such as increased urgency, frequent urination, urgency incontinence, and urine leakage at night; and (2) emptying and voiding difficulties such as weak urinary stream, incomplete emptying, and straining while urination. These symptoms are referred to as lower urinary tract symptoms (LUTS) in literature [4]. These symptoms cause a significant personal and societal burden because of both direct medical costs and reduced functioning due to decreased quality of life (QoL) of patients and their partner [5]. As the population ages, the prevalence of LUTS increases, and additional resources will be needed to solve this growing problem [6]. Various comorbidities are also usually found to be associated with $\mathrm{BPH}$, such as cardiovascular events, diabetes mellitus, digestive disorders, and inflammation accompanied by pain [7-9].

The LUTS due to BPH are usually treated by a medical or surgical intervention. The use of nonsteroidal anti-inflammatory drugs (NSAIDs) for the management of LUTS symptoms associated with BPH is still debatable [10,11]. The use of medication for the management of $\mathrm{BPH}$ is inversely related to the frequency of surgical intervention, which is believed to be due to the post-surgical complications associated with the surgical interventions available for BPH management [12]. Surgical procedures for BPH, such as transurethral resection of the prostate, laser vaporization of the prostate, and laser enucleation of the prostate, are considered safe in cases of low prostate volume; however, many adverse outcomes may occur after these surgeries that supports the use of conservative management, especially in advanced age individuals $[13,14]$.

Prostatitis syndrome is another poorly understood condition that produces prostatitis-like symptoms (for example, pelvic pain). The prostatitis-like symptoms are highly prevalent affecting about $10 \%$ of men during their lifetime [15]. The US National Institutes of Health (NIH) has classified prostatitis syndrome into 4 major categories: I-acute bacterial prostatitis, II-chronic bacterial prostatitis, III-chronic prostatitis/ chronic pelvic pain syndrome (CP/CPPS) (this category includes two subcategories, IIIa-inflammatory CPPS and IIIb-noninflammatory CPPS), and IV-asymptomatic inflammatory prostatitis [16]. The CP/CPPS is responsible for prostatitis-like symptoms in most patients (>90\%). Researchers have proposed that the chronic inflammatory processes in the prostate may play a role in the development of BPH [17]. Many researchers think that this syndrome may have multifactorial causes such as infection, inflammation, central nervous system changes, alteration in the microbiome of the urinary system, stress, and central sensitisation.

Thus, the typical treatment for it is a multimodal approach based on phenotyping, including medical intervention (alpha-blockers, antibiotics, anti-inflammatory medication, hormonal therapy, phytotherapy, and antispasmodics) and nonmedical strategies, such as psychotherapy and attempts to improve relaxation of the pelvic floor $[18,19]$. The management of $\mathrm{CP} / \mathrm{CPPS}$ is frustrating for both the patient and physician since standard treatments often fail [20] and as there has been no successful treatment available for this health problem [21]. Therefore, there has been a need in a treatment modality is needed for chronic prostatic diseases, like CP/CPPS and BPH, which is free from serious side-effects and easy to use at home by the elderly population. 
Thermobalancing therapy (TT) is a term used for elucidating the mechanism of action of the device known as therapeutic Dr Allen's device (DATD) [22, 23]. The device has been invented and used for over 10 years in people with various chronic internal diseases, including BPH and CP/CPPS [24]. The device was registered with the Medicines and Healthcare Products Regulatory Agency in the UK in 2010, as a class 1 medical device (medical device without a measuring function and supplied in a non-sterile condition and does not require the involvement of a notified body).

The DATD has been patented in the USA as a "Therapeutic device and method." The patent is an acknowledgment that it is indeed a completely new treatment option for chronic internal diseases [25]. The TT enabled by DATD facilitates the treatment of an affected area by the topical application of a special thermoelement, consisting of a mixture of waxes that accumulates the naturally emitted body heat and projects the accumulated heat to the affected body part. The device comprises an elastic belt with a pocket that houses the thermoelement and maintains it in the projection of the affected body part. Thus, TT with DATD is the first ever non-invasive treatment that employs body energy for the treatment of chronic diseases. It should be used for a prolonged period of time, ranging from days to months [26, 27].

This study aims to assess the effectiveness of this innovative therapy uniquely employing body energy for the management of chronic prostatic conditions, such as $\mathrm{BPH}$ and $\mathrm{CP} / \mathrm{CPPS}$.

\section{Methods}

This is a retrospective analysis of data obtained from 2 clinical investigations that were registered at the World Health Organization via the German Clinical Trials Register: DRKS00009271 - Effect of the Physiotherapeutic Device compared to no treatment on prostate volume, urinary symptoms, and quality of life in men with Benign Prostatic Hyperplasia (BPH), and DRKS00009352 - Effect of the physiotherapeutic device and thermobalancing therapy compared with no therapy on pain, urinary symptoms, quality of life and prostate volume in men with chronic prostatitis/chronic pelvic pain syndrome (CP/CPPS).

The ethics committee of the Yerevan State Medical University approved these 2 studies. The 2 clinical investigations included 124 men with $\mathrm{BPH}$ and 45 men with $\mathrm{CP} /$ CPPS all with age above 50 years and who were naive for the intended treatment (TT). These study participants constituted the treatment group and received TT for a period of 6 months. The TT was administered with the help of DATD applied to project a single thermoelement over the prostate gland (as shown in Fig. 1).

The results of the treatment groups were compared with those of the control groups comprising the same number of age-matched participants (124 men with BPH and 45 men with CP/CPPS) who were in the control groups of participants who were either on watchful waiting or did not receive any treatment.

Various baseline evaluations were carried out, which included complete physical examination, medical history, digital rectal examination, serum biochemistry, prostatespecific antigen measurements, electrolytes, urine examination, and renal function tests. The efficacy of TT was evaluated by using the following parameters that were measured 


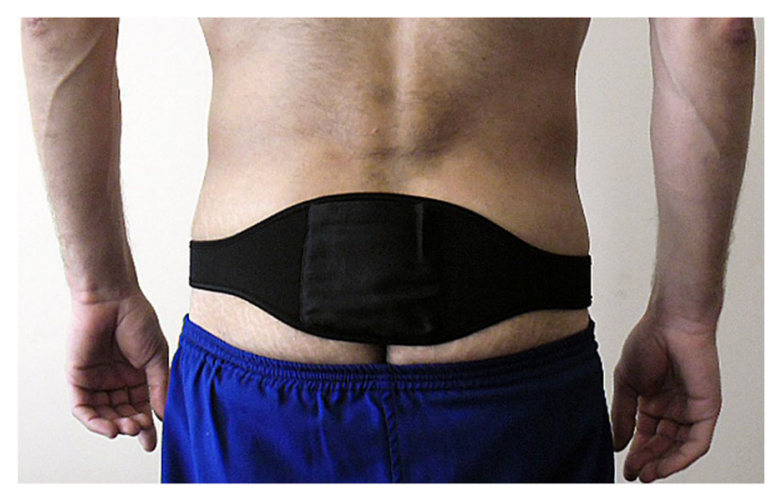

Fig. 1 Dr Allen's Device for prostate treatment comprises an elastic belt and one thermoelement

before starting the treatment and after completing the 6-month treatment (in the treatment groups) and no treatment (in the control groups):

1. The International Prostate Symptom Scale (I-PSS) is a validated and widely accepted tool that is used to assess the dynamics of urinary symptoms and quality of life (QoL) in BPH patients. The I-PSS consists of an 8-item questionnaire that measures urinary symptoms (seven questions evaluating incomplete bladder emptying, frequency, intermittency, urgency, weak stream, straining, and nocturia) on a scale of 0-35 with a higher score indicating higher severity of the urinary symptoms. The I-PSS consists of another component (1 question) that measures QoL on a scale of 0-6 with a lower score indicating a better quality of life.

2. The National Institutes of Health chronic prostatitis symptom index (NIH-CPSI) is a validated and widely accepted tool that is used to assess the dynamics of pain and QoL in CP/CPPS patients. The NIH-CPSI assesses pain using 4 questions (evaluating pain location, frequency, and severity) on a scale of 0-21 with a higher score indicating more severe pain. The NIH-CPSI consists of another component that helps in assessing QoL using 3 questions on a scale of $0-12$ with a lower score indicating a better quality of life.

3. Prostate volume (PV; in $\mathrm{mL}$ ) was measured using ultrasonography. The standard ellipsoid formula length $\times$ width $\times$ height $\times 0.52$ was used to determine the prostate volume.

The results obtained for the treatment group participants who received TT were compared with those of control group participants who did not receive any treatment. The Wilcoxon signed-rank test was employed for the comparison of results between the treatment group and the control group. $P<0.05$ was considered significant. Statistical analyses were carried out using SPSS version 22 software (IBM, Armonk, NY, USA).

\section{Results}

Impact of TT with DATD on urinary symptoms and QoL measured by I-PSS score

In the treatment group comprising 124 subjects with $\mathrm{BPH}$ who received $\mathrm{TT}$ using DATD, the urinary symptoms (UrS) decreased from 14.2 to 4.95 as measured by I-PSS 
score, while an increase in the urinary symptoms from 13.15 to 14.27 as measured using I-PSS was observed in the control group also comprising 124 subjects with BPH who did not receive any treatment. Similar results were obtained for the IPSS-QoL score that decreased from 3.9 to 1.49 in the treatment group but increased from 3.42 to 3.83 in the control group. The comparison of results between the 2 groups indicated that there was a statistically significant difference $(P<0.001)$ in the results obtained for the 2 groups (Fig. 2). Thus, the results indicate that DATD significantly decreases urinary symptoms and improves QoL in patients with $\mathrm{BPH}$, while watchful waiting may worsen the urinary symptoms and QoL in this patient population.

\section{Impact of $T T$ with DATD on pain and QoL measured by NIH-CPSI}

In another treatment group comprising 45 subjects with CP/CPPS who received TT using DATD, the pain score decreased from 10.38 to 3.58 as measured by NIH-CPSI questionnaire, while a marginal decrease in the pain score from 10.49 to 9.71 as measured using NIH-CPSI was observed in the control group also comprising 45 subjects with CP/CPPS who did not receive any treatment. Similar results were obtained for the QoL score that decreased from 8.11 to 2.98 in the treatment group but only marginally decreased from 8.47 to 8.33 in the control group. The comparison of results between the 2 groups indicated that there was a statistically significant difference $(P<0.001)$ in the results obtained for the 2 groups (Fig. 3). Thus, the results indicate that DATD significantly decreases pain and improves QoL in patients with CP/CPPS, while no treatment may not be effective in this patient population.

\section{Impact of $T T$ with DATD on prostate volume (PV)}

An increase in the PV from 45.5 to $50.85 \mathrm{~mL}$ was noted in the control group comprising 124 subjects with $\mathrm{BPH}$ who did not receive any treatment. However, there

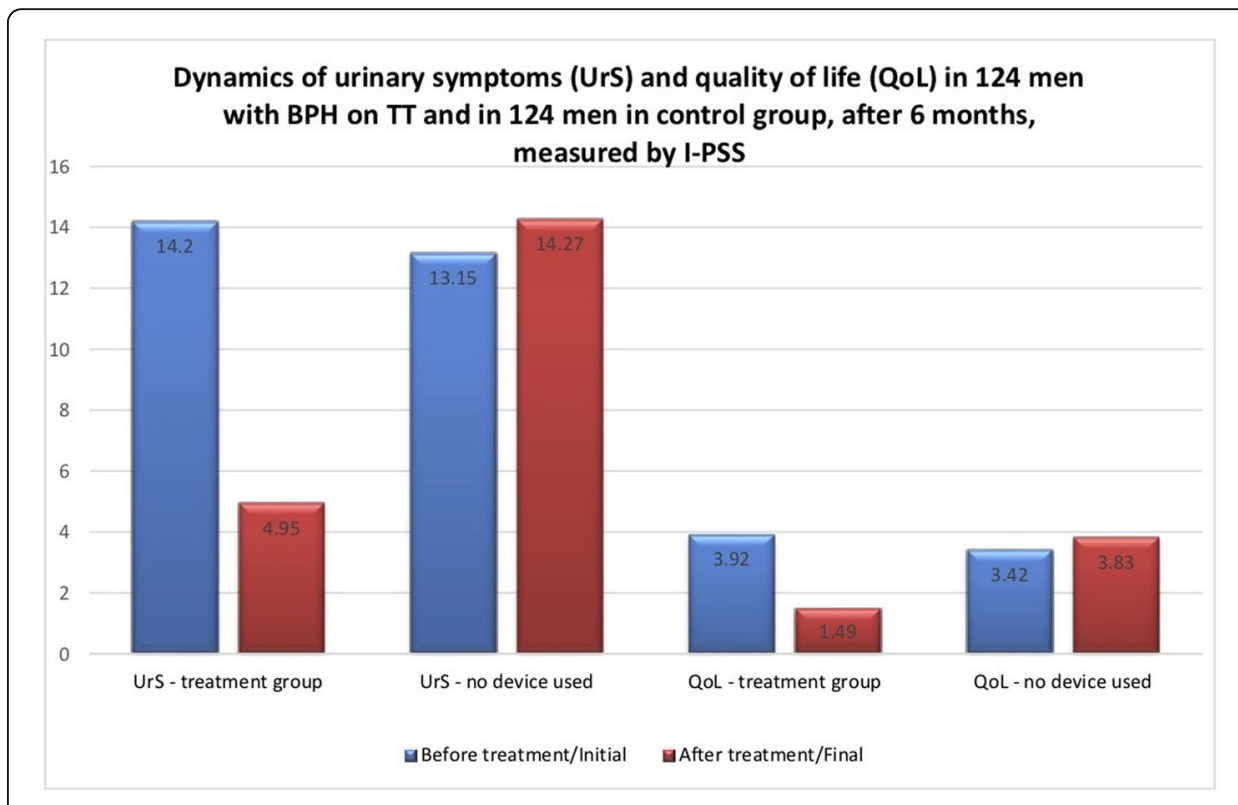

Fig. 2 Urinary symptoms (UrS) and QoL in the treatment group and control group as measured by I-PSS 


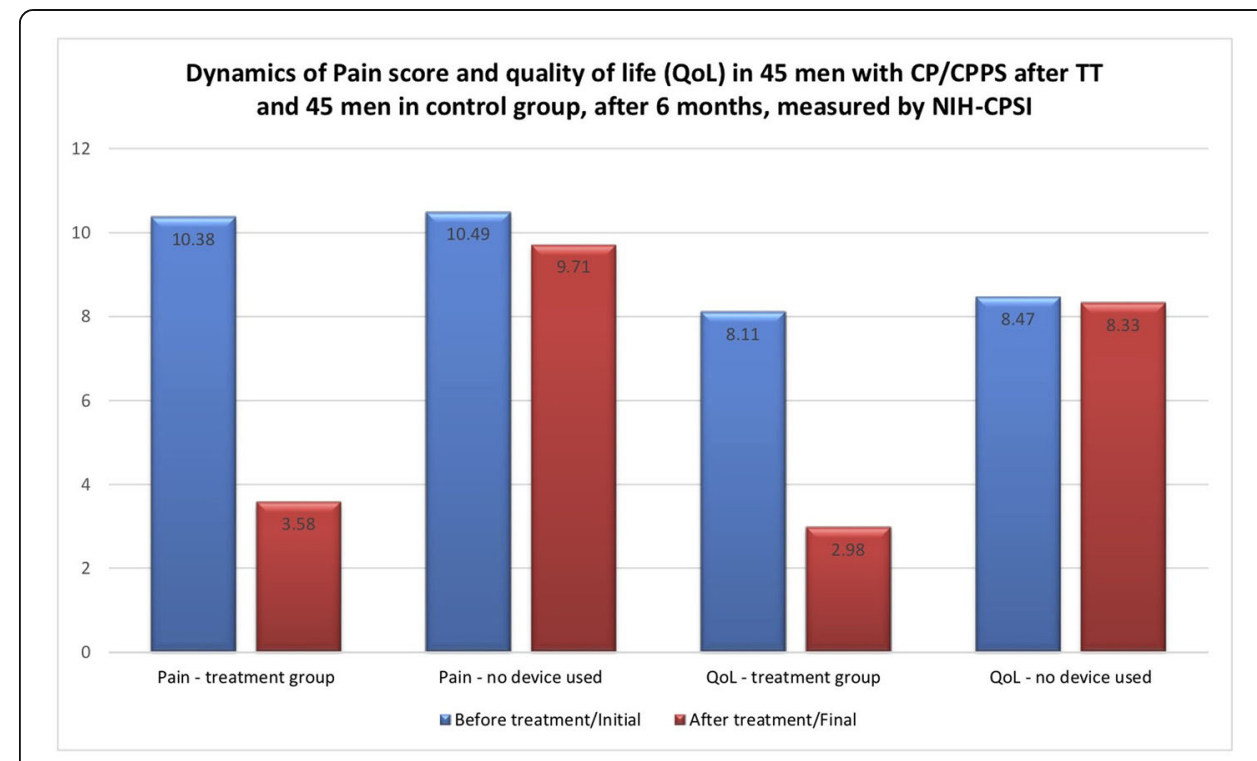

Fig. 3 Pain score and QoL in the treatment group and control group as measured by NIH-CPSI

was a marked decrease in the PV from 45.1 to $31.8 \mathrm{~mL}$ in the treatment groups comprising 124 subjects with BPH. The PV change in the treatment group was statistically significantly different when compared with that of the control group $(P<$ 0.001). Thus, the results indicate that DATD significantly decreases prostate volume (PV) in patients with $\mathrm{BPH}$, while watchful waiting may not be effective in this patient population. Similarly, in the treatment group comprising 45 men with CP/ CPPS who received TT, PV reduced from 31.7 to $27.0 \mathrm{~mL}$; however, in the control group comprising 45 men with CP/CPPS who did not receive any treatment, PV increased from 30.7 to 31.5. The comparison of results among the two CP/CPPS groups indicated a statistically significant difference between the results obtained for the two groups $(P<0.001)$. Thus, the results indicate that DATD significantly decreases prostate volume (PV) in patients with $\mathrm{CP} / \mathrm{CPPS}$, while no treatment may not be effective in this patient population.

Overall, the results indicate that the treatment with DATD reduces prostate volume and LUTS in men with $\mathrm{BPH}$ and decreases pain and prostate volume in men with $\mathrm{CP} /$ CPPS. Also, QoL improved significantly in both treatment groups, while there were no positive changes in the control groups.

\section{Discussion}

Historically, BPH was considered an outcome of the aging process and treatment modalities were devised merely to reduce the symptoms of the diseases with the help of different medicines or surgical interventions. However, developments in the last decade have indicated that vasculature plays an important role in the pathophysiology and etiology of this chronic disease [28]. Physiologists have found that constriction and spontaneous expansion of capillaries occur $[29,30]$. These processes are responsible for the build-up of pressure inside the prostate that results in abnormal growth of the prostate tissue. The constriction of capillaries in response to different triggers such as infection, cold, excess of certain elements, stress, and others creates focal hypothermia in the 
prostate tissue. To improve blood circulation in the affected area, spontaneous expansion of a capillary net occurs. The expansion of the capillaries, known as angiogenesis, slowly forms additional tissue, which creates pressure in this area and the prostate gland continues to grow in size [31]. These findings support the fact that etiology and pathophysiology of $\mathrm{BPH}$ are associated with prostate hypoxia, pelvic ischemia, increased pressure inside the prostate, and vascular dysfunction [32-35].

Similarly, the etiology and pathophysiology of CP/CPPS can be viewed as a chain of events in which constriction of capillaries in the prostate tissue, due to different initial triggers (such as cold, infection, and stress) leads to spontaneous capillary expansion. This leads to increased pressure in the prostate gland that sets up a secondary trigger, namely the focal hypothermia [36]. Chronic ischemia and vascular dysfunction in the prostate tissue has already been indicated to be involved in the pathophysiology of $\mathrm{BPH}$ and CP/CPPS [37, 38]. Also, the development of pain in men with CP/CPPS has been reported to occur due to increased intra-prostatic pressure [39]. Constant pressure in the prostate gland due to the disturbance in the prostate vasculature leads to chronic pelvic pain [40].

The current study highlights the effectiveness of innovative employment of body energy with DATD and TT in the treatment of chronic prostatic diseases, as it reduces PV and improves QoL in men with BPH and CP/CPPS. It was demonstrated that effective local energy management with DATD leads to improvement in the blood circulation within the prostate gland; consequently, a decrease in PV was observed accompanied by a reduction in the urinary symptoms in men with BPH and a reduction in the pain score in men with CP/CPPS. The studies on TT with DATD in men with $\mathrm{BPH}$ and $\mathrm{CP} / \mathrm{CPPS}$ have demonstrated that $\mathrm{TT}$ is effective because it treats the actual cause of these disorders, and since the cause of these chronic diseases is identical, they should be treated in the same way [41, 42]. DATD helps in improving blood circulation in the prostate gland due to its constant exposure to the optimal amount of heat accumulated by the thermoelement from the body's own radiated heat or energy, and no external heat source is used by the device. This is a novel mechanism of action.

The innovative approach for treating chronic prostate diseases with non-invasive $\mathrm{Dr}$ Allen's Device by improving blood circulation locally is important. Although there are many medical options for treating $\mathrm{BPH}$, their safety and effectiveness against older people are still under consideration [43]. For instance, silodosin, known as Rapaflo in the US, was approved by the FDA for BPH in 2008 [44] and recommended for men with CP/CPPS in 2011 [45]. However, even though the efficacy of silodosin appears to be similar to that of other alpha-blockers (tamsulosin and alfuzosin), its rate of sexual side effects is likely higher [46]. Moreover, a study published in 2020 suggests that silodosin was associated with increased digestive symptoms such as diarrhea and loose stools [47]. Therefore, oral drugs for chronic prostate diseases need to be selected by taking into consideration the possibility of their side effects.

The efficacy of Dr Allen's Device for the treatment of BPH and CP/CPPS has confirmed that the heat emitted by the body can serve as a source of energy, which, when directed at the prostate over a long period of time, gradually eliminates the focal hypothermia and thus alleviates LUTS symptoms of BPH and pain associated with $\mathrm{CP} /$ CPPS. Thus, TT removes a permanent trigger in the tissue of the prostate gland, termed focal hypothermia, which is responsible for chronic pelvic pain and continuous 
prostate growth. This improves the health and well-being of men [48]. The positive changes in the prostate gland, absence of any side effects, and improved QoL achieved through this innovative treatment modality make it more attractive compared with other treatment modalities. The use of DATD as a single self-management tool for the at home treatment of $\mathrm{BPH}$ and $\mathrm{CP} / \mathrm{CPPS}$ can significantly reduce the need for other interventions, reduces contact with healthcare providers and patient visits to clinics and hospitals, which is particularly important during a pandemic and cost-effective for both the patients and the society $[49,50]$.

\section{Limitation}

This is a retrospective analysis of 2 non-randomized clinical trials. The presence of a "placebo" or "sham" group as control could have provided more statistical rigor with respect to results. However, the results of a cohort study showed a bi-directional relationship between controlled anxiety/depression and LUTS due to BPH [51], and many researches since 1995 are viewing CP and/or CPPS as a psychoneuromuscular disorder driven by protective pelvic floor guarding and psychosocial stress [52]. Thus, most men with CP/CPPS and BPH have psychological problems, including depression and anxiety $[53,54]$. A 6-month period may be considered an appropriate time for taking tablets/ placebo, but not for using something tied to the body. Therefore, suggesting that patients should wear a "placebo-belt" for 6 months, which does not alleviate the symptoms, would be very difficult. Typically, patients included in the study felt relief of symptoms several days or weeks after wearing DATD and continued using the device as needed.

\section{Conclusion}

Innovative TT with DATD is an effective treatment modality for the management of chronic diseases of internal organs, including BPH and CP/CPPS. The pathophysiology of $\mathrm{BPH}$ and $\mathrm{CP} / \mathrm{CPPS}$ is related to vascular changes at the level of capillaries that lead to the development of focal hypothermia, spontaneous expansion of capillaries, and pressure in the affected tissue, which in turn leads to chronic prostate disease. TT with DATD is the first treatment modality of its kind that can effectively treat the capillary changes employing body energy and, thus, it can be used as a personalised care method that improves wellbeing naturally and cost-effectively. Moreover, this at home treatment with DATD reduces the number of patient hospital visits and contact with healthcare providers, minimising the risk of infection transmission during a pandemic. Additional clinical trials should be conducted to assess this innovative treatment modality in comparison with other standards of care approved for the treatment of chronic diseases, like BPH and CP/CPSS.

Abbreviations

TT: Thermobalancing therapy; DATD: Therapeutic Dr Allen's Device; BPH: Benign prostatic hyperplasia; CP/ CPPS: Chronic prostatitis/chronic pelvic pain syndrome; $n$ : Number; QoL: Quality of life; PV: Prostate volume; LUTS: Lower urinary tract symptoms; I-PSS: International Prostate Symptom Scale; NIH-CPSI: National Institutes of Health chronic prostatitis symptom index; US: United States; NSAIDs: Nonsteroidal anti-inflammatory drugs; UrS: Urinary symptoms 


\section{Author's contributions}

SA made a significant contribution to the concept and design of the studies. SA analysed and interpreted patient data and wrote the manuscript. SA approved the final manuscript.

\section{Funding}

The studies do not have financial interest.

\section{Availability of data and materials}

Data available at the public World Health Organization International Clinical Trials Registry http://apps.who.int/ trialsearch/Trial2.aspx?TrialID = DRKS00009271 and http://apps.who.int/trialsearch/Trial2.aspx?TriallD = DRKS00009352

\section{Competing interests}

The author declares that there are no competing interests.

Received: 5 November 2019 Accepted: 30 April 2020

Published online: 09 June 2020

\section{References}

1. Egan KB (2016) The epidemiology of benign prostatic hyperplasia associated with lower urinary tract symptoms: prevalence and incident rates. Urol Clin North Am 43:289-297. https://doi.org/10.1016/j.ucl.2016.04.001

2. Giorgio Ivan Russo Gl, Urzi D, Cimino S (2018) Epidemiology of LUTS and BPH. In Lower Urinary Tract Symptoms and Benign Prostatic Hyperplasia, Chapter 1, pp 1-14. Academic Press. https://doi.org/10.1016/B978-0-12-811397-4. 00001-9.

3. Kwong PW, Cumming RG, Chan L et al (2010) Urinary incontinence and quality of life among older communitydwelling Australian men: the CHAMP study. Age Ageing 39:349-354. https://doi.org/10.1093/ageing/afq025

4. Abdelmoteleb H, Aiello M, Drake M et al (2020) The lower urinary tract symptoms. In: Drake M., Cocci A., Pereira e Silva R. (eds) Lower urinary tract symptoms in adults. pp 19-38. Springer, Cham

5. Speakman M, Kirby R, Doyle S et al (2015) Burden of male lower urinary tract symptoms (LUTS) suggestive of benign prostatic hyperplasia (BPH) - focus on the UK. BJU Int 115:508-519

6. Zhang W, Zhang X, Li H et al (2019) Prevalence of lower urinary tract symptoms suggestive of benign prostatic hyperplasia (LUTS/BPH) in China: results from the China Health and Retirement Longitudinal Study. BMJ Open. 9(e): 022792. https://doi.org/10.1136/bmjopen-2018-022792

7. Gacci M, Corona G, Sebastianelli A et al (2016) Male lower urinary tract symptoms and cardiovascular events: a systematic review and meta-analysis. Eur Urol 70:788-796. https://doi.org/10.1016/j.eururo.2016.07.007

8. Chen Z, Miao L, Gao X et al (2015) Effect of obesity and hyperglycemia on benign prostatic hyperplasia in elderly patients with newly diagnosed type 2 diabetes. Int J Clin Exp Med 8:11289-11294

9. De Nunzio C, Presicce F, Tubaro A (2016) Inflammatory mediators in the development and progression of benign prostatic hyperplasia. Nat Rev Urol 13:613-626. https://doi.org/10.1038/nrurol.2016.168

10. Ishiguro H, Kawahara T (2014) Nonsteroidal anti-inflammatory drugs and prostatic diseases, BioMed Res. Int 436123. https://doi.org/10.1155/2014/436123

11. Kahokehr A, Vather R, Nixon A et al (2013) Non-steroidal anti-inflammatory drugs for lower urinary tract symptoms in benign prostatic hyperplasia: systematic review and meta-analysis of randomized controlled trials. BJU Int 111:304-311

12. Ingimarsson JP, Isaksson HJ, Sigbjarnarson HP et al (2014) Increased population use of medications for male lower urinary tract symptoms/benign prostatic hyperplasia correlates with changes in indications for transurethral resection of the prostate. Scand J Urol 48:73-78. https://doi.org/10.3109/21681805.2013.825878

13. Naeem B, Giorgio G, Akshay $S$ et al (2014) Morbidity and mortality after benign prostatic hyperplasia surgery: data from the American College of Surgeons National Surgical Quality Improvement Program. J Endourl 28:831-840

14. Elshal AM, Elmansy HM, Elkoushy MA et al (2012) Male sexual function outcome after three laser prostate surgical techniques: a single center perspective. Urology 80:1098-1104

15. Suskind AM, Berry SH, Ewing BA et al (2013) The prevalence and overlap of interstitial cystitis/bladder pain syndrome and chronic prostatitis/chronic pelvic pain syndrome in men: results of the RAND Interstitial Cystitis Epidemiology male study. J Urol 189:141-145. https://doi.org/10.1016/j.juro.2012.08.088

16. Rees J, Abrahams M, Doble A et al (2015) the Prostatitis Expert Reference Group (PERG). Diagnosis and treatment of chronic bacterial prostatitis and chronic prostatitis/chronic pelvic pain syndrome: a consensus guideline. BJU Int 116 509-525. https://doi.org/10.1111/bju.13101

17. Magistro G, Wagenlehner FM, Weidner GW et al (2016) Contemporary management of chronic prostatitis/chronic pelvic pain syndrome. Eur Urol 69:286-297

18. Gandaglia G, Briganti A, Gontero P (2013) The role of chronic prostatic inflammation in the pathogenesis and progression of benign prostatic hyperplasia (BPH). BJU Int 112:432-441

19. Arora HC, Eng C, Shoskes DA (2017) Gut microbiome and chronic prostatitis/chronic pelvic pain syndrome. Ann Trans Med. https://doi.org/10.21037/atm.2016.12.32

20. Benelli A, Hossain H, Pilatz A et al (2017) Prostatitis and its management. Eur Urol Suppl 16:132-137. https://doi.org/10. 1016/j.eursup.2016.03.001

21. Kessler TM (2016) Chronic pelvic pain syndrome: light at the end of the tunnel? Eur Urol 69:298-299. https://doi.org/10. 1016/j.eururo.2015.09.028

22. Allen S, Aghajanyan IG (2015) Benign prostatic hyperplasia treatment with new physiotherapeutic device. Urol J 12: 2371-2376

23. Allen S, Aghajanyan IG (2016) Effect of thermobalancing therapy on chronic prostatitis and chronic pelvic pain syndrome. Journal of Clinical Urology:1-8. https://doi.org/10.1177/2051415816671036

24. Allen S (2019) Thermobalancing therapy as self-management tool. WNNS 23:84-93 
25. Allen S, Adjani A (2016) Therapeutic device and method, United States Patent and Trademark Office. U.S. Patent 9,408,744 B2, 9 August 2016. Available online: https://www.google.com/patents/US9408744 Accessed 30 Apr 2020.

26. Allen S, Aghajanyan IG (2016) Thermobalancing conservative treatment for moderate-to-low-degree lower urinary tract symptoms (LUTS) secondary to prostate enlargement. Cogent Medicine 3:1195067 https//doi.org/10.1080/2331205x.2016.1195067

27. Allen S, Aghajanyan IG (2017) New independent thermobalancing treatment with therapeutic device for internal diseases: chronic prostatitis and chronic pelvic pain syndrome. Nephrourol Mon. 44694. https://doi.org/10.5812/ numonthly.44694

28. Allen S (2016) The origin of chronic diseases can be in capillary pathology: an evidence from clinical trials on thermobalancing treatment of prostate reveals. Achiev Life Sci 10:197-202. https://doi.org/10.1016/j.als.2016.11.005

29. Baldwin AL (2000) A brief history of capillaries and some examples of their apparently strange behaviour. Clin Exp Pharmacol Physiol 27:821-825 https://doi.org/10.1046/j.1440-1681.2000.03339.x

30. Hansen-Smith FM (2000) Capillary network patterning during angiogenesis. Clin Exp Pharmacol Physiol 27:830-835 https://doi.org/10.1046/j.1440-1681.2000.03341.x

31. Allen S, Aghajanyan IG (2016) Use of thermobalancing therapy in ageing male with benign prostatic hyperplasia with a focus on etiology and pathophysiology. Aging Male 14:1-5 https://doi.org/10.1080/13685538.2016.1247151

32. Saito M, Tsounapi P, Oikawa R et al (2014) Prostatic ischemia induces ventral prostatic hyperplasia in the SHR; possible mechanism of development of BPH. Sci Rep 4:3822. https://doi.org/10.1038/srep03822

33. Thurmond P, Yang JH, Azadzoi KM (2016) LUTS in pelvic ischemia: a new concept in voiding dysfunction. Am J Physiol Renal Physiol 310:F738-F743. https://doi.org/10.1152/ajprenal.00333.2015

34. Cohen PG (2012) Abdominal obesity and intra-abdominal pressure: a new paradigm for the pathogenesis of the hypogonadalobesity BPH-LUTS connection. Horm Mol Biol Clin Investig 11:317-320 https:/doi.org/10.1515/hmbci-2012-0030

35. Shimizu S, Tsounapi P, Shimizu T et al (2014) Lower urinary tract symptoms, benign prostatic hyperplasia/benign prostatic enlargement and erectile dysfunction: are these conditions related to vascular dysfunction? Int J Urol 21:856864 https://doi.org/10.1111/iju.12501

36. Allen S (2018) The cause, aetiology and physiopathology of chronic prostatitis/chronic pelvic pain syndrome (CP/CPPS) and its effective treatment with thermobalancing therapy. Med Sur Urol 7:207. https://doi.org/10.4172/2168-9857.1000207

37. Fujii S, Yamashita S, Hayashi N et al (2019) Phosphodiesterase type 5 inhibitor attenuates chronic ischemia-induced prostatic hyperplasia in a rat model. Prostate. 79(5):536-543. https://doi.org/10.1002/pros.23759

38. Shoskes DA, Prots D, Karns J et al (2011) Greater endothelial dysfunction and arterial stiffness in men with chronic prostatitis/ chronic pelvic pain syndrome-a possible link to cardiovascular disease. J Urol 186:907 https://doi.org/10.1016/j.juro.2011.04.063

39. Mehik A, Leskinen MJ, Hellström P (2003) Mechanisms of pain in chronic pelvic pain syndrome: influence of prostatic inflammation. World J Urol 21:90-94 https://doi.org/10.1007/s00345-003-0334-3

40. Allen $S$ (2017) The vascular factor plays the main role in the cause of pain in men with chronic prostatitis and chronic pelvic pain syndrome: the results of clinical trial on thermobalancing therapy. Diseases 5:25. https://doi.org/10.3390/ diseases 5040025

41. Allen S (2019) The cause of chronic prostatitis and prostate enlargement is a change in the vascular level and requires similar treatment: innovative Thermobalancing therapy. IJMHS 9:290-293. https://doi.org/10.15520/ijmhs.v9i2.2453

42. Aghajanyan IG, Allen S (2016) Positive response to thermobalancing therapy enabled by therapeutic device in men with nonmalignant prostate diseases: BPH and chronic prostatitis. Diseases 4:18. https://doi.org/10.3390/diseases4020018

43. Bortnick E, Simma-Chiang V, Omidele O et al (2019) Medical therapies for treatment of BPH: special considerations in elderly men. Curr Geri Rep 8:310 https://doi.org/10.1007/s13670-019-00299-1

44. Cantrell MA, Bream-Rouwenhorst, HR, Hemerson P \& Magera, JS (2010) New drug approvals: silodosin for benign prostatic hyperplasia. Ann Pharmacother 44:302-310. https://doi.org/10.1345/aph.1 M320

45. Nickel JC, O'Leary MP, Lepor $\mathrm{H}$ et al (2011) Silodosin for men with chronic prostatitis/chronic pelvic pain syndrome: results of a phase II multicenter, double-blind, placebo controlled study. J Urol 186:125 https://doi.org/10.1016/j.juro.2011.03.028

46. Jung JH, Kim J, MacDonald R et al (2017) Silodosin for the treatment of lower urinary tract symptoms in men with benign prostatic hyperplasia. Cochrane Database of Systematic Reviews Issue 11. Art. No.: CD012615. doi: https://doi. org/10.1002/14651858.CD012615.pub2.

47. Matsumoto S, Kasamo S, Hashizume K (2020) Influence of alpha-adrenoceptor antagonists therapy on stool form in patients with lower urinary tract symptoms suggestive of benign prostatic hyperplasia. Low Urin Tract Symptoms 12: 86-91. https://doi.org/10.1111/luts.12289

48. Allen S (2018) Innovative Thermobalancing therapy can help millions of men with enlarged prostate gland to improve the quality of life and well-being throughout the world. World Sci News 105:51-61.

49. Allen S (2018) Dr Allen's therapeutic devices should be Implemented in the healthcare system for the treatment of chronic noncancerous prostate and kidney diseases saving people's well-being and money. Ann Mil Health Sci Res 16: e81033. https://doi.org/10.5812/amh.81033.

50. Allen S (2019) Personalized care using thermobalancing therapy can help men with chronic prostatitis / chronic pelvic pain syndrome to recover. Per Med Universe 8:48-52. https://doi.org/10.1016/j.pmu.2019.04.002.

51. Huang CL, Wu MP, Ho CH, Wang JJ (2017) The bidirectional relationship between anxiety, depression, and lower urinary track symptoms: a nationwide population-based cohort study. J Psychosom Res 100:77-82. https:/doi.org/10.1016/j.jpsychores.2017.07. 008

52. Anderson RU, Wise D, Nathanson BH (2018) Chronic prostatitis/chronic pelvic pain as a psycho-neuromuscular disordera meta-analysis. Urology 120:23-29. https://doi.org/10.1016/j.urology.2018.07.022.

53. Chung SD, Lin HC (2013) Association between chronic prostatitis/chronic pelvic pain syndrome and anxiety disorder: a population-based study. PLoS One 8:e64630.

54. Martin S, Vincent A, Taylor AW, et al. (2015) Lower urinary tract symptoms, depression, anxiety and systemic Inflammatory factors in men: a population-based cohort study. PLoS One 10:e0137903.

\section{Publisher's Note}

